\title{
BIOACESSIBILIDADE DE ZINCO, CÁLCIO E FÓSFORO EM EXTRATO DE SOJA E AMOSTRAS DE LEITE BOVINO, CAPRINO E OVINO
}

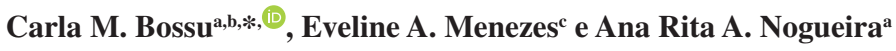 \\ ${ }^{a}$ Embrapa Pecuária Sudeste, 13560-970 São Carlos - SP, Brasil \\ bDepartamento de Química, Universidade Federal de São Carlos, 13560-970 São Carlos - SP, Brasil \\ 'Universidade da Integração Internacional da Lusofonia Afro-Brasileira, Campus das Auroras, 62790-970 Redenção - CE, Brasil
}

Recebido em 14/01/2020; aceito em 25/03/2020; publicado na web em 05/05/2020

\begin{abstract}
BIOACESSIBILITY OF ZINC, CALCIUM AND PHOSPHORUS IN SOYBEAN EXTRACT AND COW, GOAT, SHEEP MILK SAMPLES. This work describes the bioaccessibility in vitro simulated gastric digestion for levels of $\mathrm{Zn}, \mathrm{Ca}$, and $\mathrm{P}$ in milk and soybean extract samples. Total and dialyzed levels were determined by ICP OES. According to results of the dialyzed samples, it can be concluded that in $250 \mathrm{~mL}$ of milk (a glass) the body can be absorb about $1.2 \pm 0.2 \mathrm{mg}, 14.8 \pm 1.2 \mathrm{mg}, 4.5 \pm 0.8 \mathrm{mg}, 2.2 \pm 0.4 \mathrm{mg}$ and $4.9 \pm 0.6 \mathrm{mg}$ of $\mathrm{Zn} ; 2079 \pm 133 \mathrm{mg}, 305 \pm 4 \mathrm{mg}, 2396 \pm 16 \mathrm{mg}, 2444 \pm 54 \mathrm{mg}$, and $1527 \pm 71 \mathrm{mg}$ of Ca; and $2023 \pm 275 \mathrm{mg}$, $811 \pm 14 \mathrm{mg}, 2688 \pm 39 \mathrm{mg}, 5089 \pm 474 \mathrm{mg}$ and $3467 \pm 63 \mathrm{mg}$ of P for raw sheep's milk, soybean, raw cow's milk, UHT goat's milk, and UHT cow's milk, respectively. These results correspond to approximately 45,118 , and $267 \%$ of diary human needed of $\mathrm{Zn}, \mathrm{Ca}$, and P, respectively. Accuracy was verified using CRM (NIST 8435) for total levels, and dialyzed samples were compared with FAAS, statistically evaluated by Student's $t$-test. Results confirmed the methodology efficiency and allowed an estimate of the bioacessibility of nutrients.
\end{abstract}

Keywords: zinc; calcium; phosphorus; milk; bioaccessibility.

\section{INTRODUÇÃO}

A estimativa da biodisponibilidade, isso é, a proporção de um dado nutriente em um dado alimento ou dieta que o corpo realmente utilize e a bioacessibilidade, que se refere a "fração de um composto que é liberado de sua matriz no trato gastrointestinal e, assim, torna-se disponível para absorção intestinal (ou seja, entra na corrente sanguínea)", ${ }^{1,2}$ juntamente com a determinação das formas químicas relacionadas às fontes de minerais utilizados são importantes, considerando que os elementos normalmente não se encontram totalmente disponíveis do ponto de vista nutricional ou ambiental. Os minerais podem se apresentar na forma inorgânica (óxidos, sulfatos, carbonatos, etc), ou na forma orgânica, sendo que geralmente as fontes inorgânicas apresentam baixa biodisponibilidade. ${ }^{3,4}$

Do ponto de vista nutricional, é de grande interesse o conhecimento dos teores de zinco $(\mathrm{Zn})$, cálcio $(\mathrm{Ca})$ e fósforo $(\mathrm{P})$ no leite e as frações desses elementos que podem ser absorvidas ou utilizadas, ou seja, a sua bioacessibilidade. ${ }^{5} \mathrm{~A}$ abordagem ideal seria a avaliação in vivo, porém esses estudos são, em geral, demorados e até impraticável para aplicações em grande escala. ${ }^{6}$

Os métodos in vitro constituem uma boa alternativa para os métodos in vivo na avaliação da bioacessibilidade de nutrientes, sendo assim, os mais estudados. Esses métodos normalmente se baseiam na simulação da digestão gastrointestinal, seguida pela determinação da quantidade de um determinado elemento solúvel ou dialisado através de uma membrana de certo tamanho dos poros. ${ }^{7,8}$

Peptídeos fosforilados (CPPs), codificados como $\alpha_{s 1^{-}}, \alpha_{s 2^{-}}$e $\beta$-caseínas podem formar complexos solúveis com minerais como cálcio, ferro e zinco em pH intestinal, modulando sua bioacessibilidade, possibilitando sua ação como minerais solúveis ou transportadores, conhecidos como caseínas fosfopeptídicas (CPPs) que podem ser liberadas durante a digestão enzimática de produtos derivados do leite. ${ }^{9,10}$ Dessa forma, com o conhecimento das principais

*e-mail: carla.bossu@gmail.com formas químicas é possível se estudar a bioacessibilidade de um dado elemento, sendo possível aprimorar, por exemplo, a dieta dos animais para a produção de alimentos mais indicados para as populações.

Existe uma grande demanda por procedimentos que possibilitem a determinação da concentração, da forma química e do valor nutritivo de elementos traço nos alimentos, os quais podem viabilizar a possibilidade de se obter informações relevantes a respeito da ligação metal-proteína. O desenvolvimento de metodologias analíticas exatas e seletivas para a quantificação dessas diferentes espécies é de extrema importância. ${ }^{5,11}$

Nesse contexto, o objetivo deste trabalho foi estimar a bioacessibilidade e redistribuição de $\mathrm{Zn}$, Ca e P nas amostras de leite bovino integral in natura, ovelha in natura, bovino integral UHT, cabra integral UHT e extrato de soja após a simulação in vitro da digestão gastrointestinal.

\section{PARTE EXPERIMENTAL}

\section{Instrumentação}

Espectrômetro óptico de emissão com plasma acoplado indutivamente (ICP OES) com configuração radial (Varian, Vista RL, Mulgrave, Austrália) foi empregado para a determinação dos teores totais e os digeridos in vitro de $\mathrm{Zn}, \mathrm{Ca}$ e P. Nesse equipamento foi empregado nebulizador V-Groove, câmara de nebulização Sturman Master, potência da rádio frequiência de $1,05 \mathrm{~kW}$. As vazões do gás no nebulizador, do plasma e do gás auxiliar foram $0,60 \mathrm{~L} \mathrm{~min}^{-1}$, $15 \mathrm{~L} \mathrm{~min}^{-1}$ e $1,50 \mathrm{~L} \mathrm{~min}^{-1}$, respectivamente. Os comprimentos de onda foram otimizados, sendo utilizados $213,857 \mathrm{~nm}$ para o $\mathrm{Zn}$, 396, $847 \mathrm{~nm}$ para Ca e 178, $222 \mathrm{~nm}$ para $\mathrm{P}$.

\section{Reagentes e soluções}

Todos os materiais empregados foram previamente lavados com detergente neutro e água corrente, abundantemente enxaguados com 
água destilada e deixados em banho de $\mathrm{HNO}_{3} 10 \%$ (v/v) por no mínimo $24 \mathrm{~h}$. A seguir, os materiais foram enxaguados com água ultrapura.

Todo o preparo de soluções, os procedimentos de preparo de amostras e bioacessibilidade foram feitos em capela de fluxo laminar para evitar problemas de contaminação.

Reagentes de grau analítico e água deionizada a $18 \mathrm{M} \Omega \mathrm{cm}$ em sistema Milli-Q ${ }^{\circledast}$ (Millipore, Bedford, MA, USA) foram empregados para preparo das soluções. As soluções estoque de Zn, Ca e P foram preparadas a partir da diluição de solução padrão comercial $1000 \mathrm{mg} \mathrm{L}^{-1}$ (Teclab, Brasil). As curvas de calibração para as determinações de teores totais em meio $\mathrm{HNO}_{3}$ (Aldrich Chemical, Milwaukee, WI, USA) $1 \%$ (v/v) e dos teores dialisados em meio $0,1 \mathrm{~mol} \mathrm{~L}^{-1}$ de $\mathrm{NaHCO}_{3}$ (Vetec Química Fina Ltda., Brasil) foram de 10 a $200 \mathrm{mg} \mathrm{L}^{-1}$ para Ca e P e de 0,1 a $2 \mathrm{mg} \mathrm{L}^{-1}$ para Zn.

\section{Amostras e teores totais}

Amostras de leite puro de vacas Holandesas e ovelha Santa Inês foram coletadas na Embrapa Pecuária Sudeste, localizada em São Carlos, SP, enquanto amostras de leite de vaca UHT, leite de cabra UHT e extrato a base de soja foram adquiridas no mercado local de São Carlos, SP, Brasil. As amostras foram armazenadas em geladeira a $-4^{\circ} \mathrm{C}$ antes das análises e o material de referência certificado utilizado para avaliar a exatidão do método proposto foi o National Institute of Science and Technology (NIST, Gaithersburg, MD, USA) Whole Milk Powder (NIST 8435).

Os teores totais de $\mathrm{Zn}$, Ca e P foram determinados nas amostras empregando forno com radiação micro-ondas com cavidade (Multiwave, Anton Parr) contendo rotor com 6 frascos de polietileno modificado. Basicamente, 2,5 mL das amostras de leite ou $250 \mathrm{mg}$ de material certificado de referência (NIST 8435 Whole Milk Powder), foram utilizados na determinação. Assim, $1,0 \mathrm{~mL}$ de $\mathrm{H}_{2} \mathrm{O}_{2}$ (Aldrich Chemical, Milwaukee, WI, USA) $(30 \% \mathrm{~m} / \mathrm{m})$ e $2,0 \mathrm{~mL} \mathrm{HNO}_{3}$ $(50 \% \mathrm{v} / \mathrm{v})$ foram utilizados nas digestões. O programa de aquecimento utilizado foi de 5 min de 0 a $100 \mathrm{~W}, 5 \mathrm{~min}$ a $600 \mathrm{~W}, 5 \mathrm{~min}$ a $1000 \mathrm{~W}$ seguido por 15 min de resfriamento. Após a digestão, as amostras foram diluídas para $20 \mathrm{~mL}$ com água deionizada e as frações de massa foram determinadas por ICP OES.

\section{Estudos de bioacessibilidade in vitro}

Para a simulação da digestão gastrointestinal in vitro usou-se potenciômetro (pH Meter Tec-2), banho-maria (Solab, Piracicaba, Brasil), mesa agitadora (Marconi, Piracicaba, Brasil) e membrana de diálise com tamanho de poros de 12000-16000 Da (Inlab, São Paulo, Brasil). A bioacessibilidade do Zn, Ca e P nas diferentes amostras estudadas foi determinada com o emprego de procedimento in vitro desenvolvido por Miller et al., ${ }^{12}$ com modificações. O experimento baseou-se na simulação da digestão gastrointestinal com pepsina- $\mathrm{HCl}$ durante a fase gástrica e sais de bile-pancreatina na fase intestinal. A solução de pepsina (Vetec Química Fina Ltda., Brasil) foi preparada dissolvendo $16 \mathrm{~g}$ de pepsina em $100 \mathrm{~mL}$ de $\mathrm{HCl}$ (Aldrich Chemical, Milwaukee, WI, USA) $0,1 \mathrm{~mol} \mathrm{~L}^{-1}$. A solução de pancreatina (Sigma-Aldrich, Germany) e sais biliares (Sigma-Aldrich, Germany) foi preparada pela dissolução de $0,5 \mathrm{~g}$ de pancreatina e $3,13 \mathrm{~g}$ de extrato de bile em $125 \mathrm{~mL}$ de $\mathrm{NaHCO}_{3} 0,1 \mathrm{~mol} \mathrm{~L}^{-1}$. Adicionaram-se $40 \mathrm{~mL}$ de cada amostra, em triplicata, e ajustou-se o $\mathrm{pH}$ para $2 \mathrm{com}$ solução de $\mathrm{HCl} 2 \mathrm{~mol} \mathrm{~L}^{-1}$. Em seguida, 6,4 $\mathrm{mL}$ de pepsina em meio $0,1 \mathrm{~mol} \mathrm{~L}^{-1}$ de $\mathrm{HCl}$ foi adicionado e a solução foi transferida para banho-maria a $37{ }^{\circ} \mathrm{C}$ com agitação $(127 \mathrm{rpm})$, permanecendo nesta condição durante 2 horas. Essa etapa simula a digestão do alimento que ocorre no estômago. Após esta etapa, cada amostra de $40 \mathrm{~mL}$ do digerido de pepsina foi separada em duplicatas de $20 \mathrm{~mL}$, sendo uma delas utilizada para o procedimento de titulação e a outra para a diálise. Para o procedimento de titulação, $5 \mathrm{~mL}$ de solução de pancreatina e sais de bile foram adicionados nos $20 \mathrm{~mL}$ do digerido de pepsina de cada amostra. Em seguida, titulou-se as amostras com $0,5 \mathrm{~mol} \mathrm{~L}^{-1}$ de solução de $\mathrm{KOH}$ (Chemco, Campinas, Brasil) até aproximadamente $\mathrm{pH} 7,5$, para simulação do valor de $\mathrm{pH}$ encontrado no meio do intestino de um indivíduo. Anotou-se o volume de $\mathrm{KOH}$ utilizado na titulação. Esse volume de $\mathrm{KOH}$ anotado foi utilizado para a preparação de $\mathrm{NaHCO}_{3}$ e, assim, colocado dentro da membrana de diálise hidratada. Dessa forma, o interior da membrana de diálise deve estar em meio tamponado $\left(\mathrm{NaHCO}_{3}\right)$ para que, durante o processo de diálise, não ocorra mudança brusca de $\mathrm{pH}$ e precipitação das proteínas. Transferiu-se $6 \mathrm{~mL}$ de solução $0,5 \mathrm{~mol} \mathrm{~L}^{-1} \mathrm{NaHCO}_{3}$ e ajustou-se o volume para $25 \mathrm{~mL}$ com água deionizada. A seguir, colocou-se essa solução dentro da membrana de diálise, que foi fechada cuidadosamente nas duas extremidades. A membrana foi colocada dentro de um becker em contato com a amostra digerida com pepsina e levado ao banho-maria por 30 minutos a $37^{\circ} \mathrm{C}$ e com $127 \mathrm{rpm}$ de agitação. Em seguida, adicionou-se $5 \mathrm{~mL}$ de solução de pancreatina e sais de bile, juntamente com a amostra e a membrana de diálise, sendo novamente colocada em banho-maria sob agitação a $127 \mathrm{rpm}$ a $37^{\circ} \mathrm{C}$ durante 2 horas. ${ }^{13}$ Após esse período, o conteúdo das membranas foi retirado e o dialisado analisado em triplicata, para a determinação de Zn, Ca e P por ICP OES. O cálculo realizado para a determinação da porcentagem dialisada (E) está apresentado na equação 1 .

$\%$ de $\mathrm{E}$ dialisável $=\frac{\mathrm{E} \text { dialisado }(\mathrm{mg} / \mathrm{L}) \times \text { volume dialisado }(25 \mathrm{~mL})}{\mathrm{E} \text { total }(\mathrm{mg} / \mathrm{L}) \times \text { volume inicial da amostra }(20 \mathrm{~mL})} \times 100$

\section{RESULTADOS E DISCUSSÃO}

\section{Determinação dos teores totais}

A composição química e a determinação dos teores de minerais no leite são importantes para o entendimento da influência fisiológica na nutrição humana e animal e a toxicidade dos elementos traço. ${ }^{14}$ Os resultados obtidos para o material de referência certificado e para as amostras estudadas estão apresentados respectivamente nas Tabelas 1 e 2 .

Os resultados obtidos para $\mathrm{Zn}$ e Ca em leite bovino (Tabela 2) se encontram na mesma faixa de concentração observada por outros autores. ${ }^{15,16} \mathrm{O}$ mesmo ocorreu com o fósforo, cujos teores obtidos estão de acordo com trabalho de Gaucheron ${ }^{16}$ e também para os três elementos em leite de cabra, de ovelha e bovino demonstrados em Raynal-Ljutovaca et al. ${ }^{17}$ Para o extrato de soja, na fase solúvel, valores similares aos obtidos neste trabalho foram observados na literatura. ${ }^{18,19}$ Como esperado, Ca apresentou os valores mais elevados, pois é o nutriente mais abundante no leite, seguido do P. Esses dois nutrientes estão bastante relacionados, apresentando uma razão $\mathrm{Ca} / \mathrm{P}$ normalmente constante e importante na dieta.

$\mathrm{O} \mathrm{Zn}$, dentre os micronutrientes presentes no leite, é o que apresenta uma maior concentração. Também se apresenta importante na

Tabela 1. Frações de massa $\left(\mathrm{mg} \mathrm{kg}^{-1}\right)$ de $\mathrm{Zn}$, Ca, e $\mathrm{P}$ obtidas em material de referência certificado de leite (NIST 8435) e coeficiente de variação $(\mathrm{CV}, \%), \mathrm{n}=3$

\begin{tabular}{cccc}
\hline & Valor certificado & Valor obtido & CV $(\%)$ \\
\hline $\mathrm{Zn}$ & $28,0 \pm 3,1$ & $30,5 \pm 0,4(0,9)$ & 1,3 \\
$\mathrm{Ca}$ & $9220 \pm 490$ & $10408 \pm 645(2,6)$ & 6,2 \\
$\mathrm{P}$ & $7800 \pm 490$ & $8604 \pm 666(2,1)$ & 7,7 \\
\hline
\end{tabular}

Valor de $t$ de Student com 95\% de confiança $=4,30 ;()=$ valor de $t$ calculado. 
nutrição humana e animal, associado às moléculas orgânicas e na forma de sais inorgânicos. No caso de aminoácidos como a histidina e a metionina, além de fosfatos e ácidos orgânicos, ajudam na biodisponibilidade deste elemento. ${ }^{20}$ Além disso, esses três elementos estão de certa forma associados a uma das mais importantes proteínas do leite, a caseína, sendo que no leite bovino o $\mathrm{Zn}$ formaria o complexo Zn-caseína-Ca-P. ${ }^{21}$

Com base nos resultados obtidos para o material de referência certificado, comparando com os valores de referência, foi realizado o teste $t$-Student não pareado e pode-se afirmar que o procedimento de preparo e determinação são adequados para a determinação dos teores totais. Deve ser observado que os valores apresentados pelo material de referência se referem à matéria seca, razão da disparidade aparente entre os valores, pois o leite apresenta aproximadamente $90 \%$ de teor de água. Assim, os três elementos em estudo ficaram a um nível de $95 \%$ de confiança, não apresentando diferenças significativas entre os resultados obtidos e os valores certificados (Tabela 1). Os limites de detecção e quantificação foram calculados conforme descrito por Thomsen et al. ${ }^{22}$ a partir de 10 medidas da solução do branco analítico considerando-se as medidas da razão do sinal analítico / sinal de fundo (SBR) e a concentração do analito que produz um sinal líquido (altura de pico) equivalente à intensidade do sinal de fundo (BEC). Os valores encontrados dos limites de detecção e quantificação foram $0,02 \mathrm{mg} \mathrm{L}^{-1}$ e $0,06 \mathrm{mg} \mathrm{L}^{-1}$ para Zn, $0,2 \mathrm{mg} \mathrm{L}^{-1}$ e $0,74 \mathrm{mg} \mathrm{L}^{-1}$ para $\mathrm{Ca}$ e $2,1 \mathrm{mg} \mathrm{L}^{-1}$ e $7,0 \mathrm{mg} \mathrm{L}^{-1}$ para $P$, respectivamente.

\section{Estudos da bioacessibilidade in vitro}

Com relação aos teores totais de $\mathrm{Zn}$, Ca e $\mathrm{P}$ nas diferentes amostras estudadas obteve-se também os teores absorvidos (Equação 1) desses elementos com o experimento in vitro. Os resultados são apresentados na Tabela 3 .

As curvas de calibração e a linearidade preparadas na presença de $\mathrm{HNO}_{3} 1,0 \%$ (v/v) e preparadas em presença de $0,1 \mathrm{~mol} \mathrm{~L}{ }^{-1}$ de $\mathrm{NaHCO}_{3}$ foram comparadas para verificação da possibilidade de interferências. Assim, as inclinações e os coeficientes de correlação dessas curvas de calibração para Ca foram, respectivamente, 165042 e 0,9992 para a curva preparada na presença de $1,0 \%$ de $\mathrm{HNO}_{3}$ e 205151 e 0,9981 para a curva preparada na presença de $0,1 \mathrm{~mol} \mathrm{~L}^{-1}$ de $\mathrm{NaHCO}_{3}$. Para P foram, respectivamente, 3,93 e 0,9987 para a curva preparada na presença de $1,0 \%$ de $\mathrm{HNO}_{3}$ e 4,23 e 0,9999 para a curva preparada na presença de $0,1 \mathrm{~mol} \mathrm{~L}^{-1}$ de $\mathrm{NaHCO}_{3}$. E para $\mathrm{Zn}$ foram, respectivamente, 1257 e 0,9993 para a curva preparada na presença de $1,0 \%$ de $\mathrm{HNO}_{3}$ e 1177 e 0,9999 para a curva preparada na presença de $0,1 \mathrm{~mol} \mathrm{~L}^{-1}$ de $\mathrm{NaHCO}_{3}$. A razão de inclinação entre as duas curvas de calibração para cada elemento foram de 1,20, 1,08 e 1,07 para Ca, P e Zn, respectivamente, não mostrando efeito significativo de $\mathrm{NaHCO}_{3}$ nas determinações.

Para avaliar a exatidão, os resultados obtidos para as amostras dialisadas de leite de ovelha in natura foram comparados com determinações realizadas por espectrometria de absorção atômica com chama (FAAS), conforme método descrito na literatura, ${ }^{23}$ especialmente para $\mathrm{Zn}$, e não apresentaram diferenças significativas pelo teste $t$ de Student, a 95\% de confiança com recuperações entre 89 e $97 \%$ dos dialisados.

Os valores encontrados dos limites de detecção e quantificação foram $0,06 \mathrm{mg} \mathrm{L}^{-1}$ e $0,2 \mathrm{mg} \mathrm{L}^{-1}$ para $\mathrm{Zn}, 0,1 \mathrm{mg} \mathrm{L}^{-1}$ e $0,23 \mathrm{mg} \mathrm{L}^{-1}$ para $\mathrm{Ca}$ e $1,0 \mathrm{mg} \mathrm{L}^{-1}$ e $3,4 \mathrm{mg} \mathrm{L}^{-1}$ para $\mathrm{P}$, respectivamente.

Os valores das porcentagens do $\mathrm{Zn}$ dialisado obtido neste estudo para as amostras de leite de bovinos foram similares aos observados por Perales et al. ${ }^{24}$ quando foi aplicado o método de digestão in vitro. Em estudos com amostras não fortificadas com cálcio, o teor de zinco absorvido foi em torno de $14 \pm 1 \%$. Por outro lado, em amostras com maiores quantidades de cálcio (fortificadas) os teores de $\mathrm{Zn}$ bioacessível (dialisado) se restringiram à faixa de $7 \mathrm{a} 8 \%$. Isso pode ser explicado pelo efeito negativo de cálcio sobre a bioacessibilidade do $\mathrm{Zn}$, em função de efeito competitivo entre os elementos, ou seja, quanto maior o teor de $\mathrm{Ca}$, menor será a bioacessibilidade do $\mathrm{Zn} .{ }^{24}$ Dessa forma, nos resultados obtidos neste trabalho, apesar das amostras estudadas não serem fortificadas com cálcio, pode-se observar também um menor teor bioacessível de Zn nos leites caprino e ovino, provavelmente em função dos maiores teores de Ca presentes nessas amostras.

Os maiores teores de $\mathrm{Zn}$ absorvidos nas amostras de leites de caprinos e ovinos $(87,5 \%$ em leite de cabra e $91,6 \%$ em leite de ovelha) foram encontradas na fração micelar. A caseína é o principal ligante do $\mathrm{Zn}$ em leite ovino, caprino e bovino. Como resultado desta distribuição, por se encontrar largamente ligado a moléculas de citrato na fração solúvel, o $\mathrm{Zn}$ se encontra mais bioacessível em leite humano. ${ }^{25}$ Os autores observaram, em estudos in vitro, $15 \%$ de bioacessibilidade de $\mathrm{Zn}$ em leite humano, sendo apenas $1 \%$ para leite de ovinos ${ }^{26}$ e, resultados similares aos aqui observados em relação às amostras de leite ovino.

Estudos in vitro ${ }^{27}$ sobre a ligação do Zn-proteínas do leite bovino para determinar os fatores que influenciariam a acessibilidade do $\mathrm{Zn}$, indicaram que em $\mathrm{pH}$ levemente alcalino, $1 \mathrm{mg}$ de caseína estaria

Tabela 2. Teores totais de $\mathrm{Zn}, \mathrm{Ca}$ e $\mathrm{P}\left(\mathrm{mg} \mathrm{L}^{-1}\right)$ obtidos nas amostras de extrato de soja e leite bovino integral UHT, bovino integral in natura, cabra integral UHT e ovelha in natura e coeficiente de variação $(\mathrm{CV}, \%), \mathrm{n}=3$

\begin{tabular}{ccccccccccc}
\hline & Soja & CV $(\%)$ & Bovino UHT & CV $(\%)$ & $\begin{array}{c}\text { Bovino } \\
\text { in natura }\end{array}$ & CV (\%) & Cabra UHT & CV (\%) & $\begin{array}{c}\text { Ovelha } \\
\text { in natura }\end{array}$ & CV (\%) \\
\hline Zn & $2,7 \pm 0,1$ & 3,7 & $3,4 \pm 0,1$ & 0,9 & $3,4 \pm 0,1$ & 2,9 & $3,22 \pm 0,02$ & 0,6 & $2,76 \pm 0,01$ & 0,4 \\
$\mathrm{Ca}$ & $153 \pm 5$ & 3,3 & $1074 \pm 6$ & 0,6 & $955 \pm 10$ & 1,0 & $1121 \pm 8$ & 0,7 & $1671 \pm 5$ & 0,3 \\
$\mathrm{P}$ & $324 \pm 9$ & 2,8 & $854 \pm 1$ & 0,1 & $709 \pm 1$ & 0,1 & $1144 \pm 18$ & 1,6 & $1575 \pm 44$ & 2,8 \\
\hline
\end{tabular}

Tabela 3. Teores bioacessíveis (\%) de Zn, Ca e P obtidos pelo método in vitro nas amostras de leite bovino integral UHT, leite bovino integral in natura, extrato de soja, leite de cabra integral UHT e leite de ovelha in natura, e coeficiente de variação (CV, \%), n = 3

\begin{tabular}{ccccccccccc}
\hline & Bovino UHT & CV $(\%)$ & $\begin{array}{c}\text { Bovino } \\
\text { in natura }\end{array}$ & CV $(\%)$ & Soja & CV $(\%)$ & Cabra UHT & CV (\%) & $\begin{array}{c}\text { Ovelha } \\
\text { in natura }\end{array}$ & CV (\%) \\
\hline Zn & $11 \pm 1$ & 9 & $11 \pm 2$ & 18,0 & $44 \pm 4$ & 8,0 & $5 \pm 1$ & 20,0 & $3,5 \pm 0,4$ & 12,0 \\
$\mathrm{Ca}$ & $12 \pm 1$ & 8,0 & $20,1 \pm 0,1$ & 1,0 & $15,9 \pm 0,2$ & 1,3 & $17,4 \pm 0,4$ & 2,2 & $10 \pm 1$ & 10,0 \\
$\mathrm{P}$ & $32 \pm 1$ & 2,0 & $30,3 \pm 0,4$ & 1,5 & $20,0 \pm 0,4$ & 1,7 & $36 \pm 3$ & 9,0 & $10 \pm 1$ & 10,0 \\
\hline
\end{tabular}


ligada a 8,4 $\mu \mathrm{g}$ de zinco. Em meio ácido do estômago, Zn é liberado a partir de caseína e absorvido no duodeno. Para recém nascidos, a absorção de Zn pode diminuir ainda mais devido à presença de zinco ligado a fosfopeptídeos resultantes da ação enzimática da tripsina e quimotripsina na caseína. ${ }^{27,28}$ Portanto, diferenças na conformação e saturação da caseína com $\mathrm{Zn}$ e outros elementos, além de diferentes condições experimentais e procedimentos de diversos trabalhos sobre esse tema relacionados aos métodos in vitro e in vivo, podem explicar algumas diferenças observadas em relação aos estudos propostos para avaliar a absorção do $\mathrm{Zn} .^{28}$

No caso do extrato de soja foi observado maior teor bioacessível de $\mathrm{Zn}(44 \pm 4 \%)$. Como a proteína da soja contém ácido fítico que interfere na absorção do $\mathrm{Zn}$, produtos alimentícios a base de soja são frequentemente considerados como pobres fontes de $\mathrm{Zn}$ disponível. ${ }^{29,30}$ Apesar disso, estudos da bioacessibilidade de $\mathrm{Zn}$ em ratos (in vivo) mostraram resultados similares aos observados neste trabalho. Para uma fórmula a base de soja, experimentos in vivo apresentaram cerca de 39,5 \pm 9,3\% de Zn bioacessível. ${ }^{5,31}$ Assim, a mera presença de proteína de soja em produtos alimentares não pode ser utilizada como o único critério para avaliar o valor nutritivo do alimento em relação ao $\mathrm{Zn}$. A bioacessibilidade de $\mathrm{Zn}$ em fórmulas à base de soja e outros alimentos pode ser influenciada por diversos fatores, incluindo os processos de transformação, fontes de proteínas e outros componentes das fórmulas nutricionais. Porém, os mesmos autores observaram elevada bioacessibilidade também em amostras de leite bovino, quando comparado ao método in vitro aqui estudado. Em ratos obteve-se $42 \pm 5,9 \%$ para leite bovino não processado (puro) e $50,9 \pm 11,1 \%$ para leite bovino processado. ${ }^{31}$

Em geral, comparando os resultados obtidos neste trabalho com os resultados de trabalhos citados utilizando métodos in vitro, pode-se confirmar grande concordância, principalmente quanto aos leites de bovinos e ovinos, indicando uma adequação do procedimento empregado.

Os valores de Ca bioacessível (Tabela 3) obtidos nesse estudo para as amostras de leite bovino foram similares aos observados na literatura. $^{24}$

Roig et al. ${ }^{7}$ estudaram amostras de leite bovino, extrato de soja, leite humano e fórmulas infantis utilizando diálise in vitro e obtiveram $20,0 \pm 0,9 \%$ como valor bioacessível para o leite bovino, sendo que para o extrato de soja obtido de três diferentes fabricantes foram encontrados os valores $18,0 \pm 0,7 \%, 12,7 \pm 1,6 \%$ e $17,6 \pm 0,9 \%$ de bioacessibilidade de $\mathrm{Ca}$, indicando resultados similares aos obtidos no presente trabalho. Os mesmos autores, ${ }^{32}$ também utilizando diálise in vitro observaram $20,2 \pm 1,4 \%$ e $13,0 \pm 1,3 \%$ respectivamente em amostras de leite bovino e fórmula a base de soja, concordando com os aqui observados para leite bovino e indicando pequena variação em relação ao extrato de soja. Além disso, autores mostraram que a bioacessibilidade de Ca nos diferentes tipos de leite (humano, bovino, cabra e ovelha), avaliados por método in vitro através de diálise, não diferiram significativamente, variando entre 18 e $23 \% .{ }^{26}$

O Ca coloidal presente no leite pode ser definido como uma mistura de caseinato de cálcio (contendo fosfato orgânico) e fosfato de cálcio (que é um fosfato inorgânico). Existem quantidades significativas de cálcio-fosfato e cálcio-citrato nas micelas de caseína. Esse fosfato de cálcio micelar, que é principalmente associada à $\alpha s_{1^{-}}, \alpha s_{2}-$ e $\beta$-caseínas, é responsável pela estabilidade e estrutura das micelas de caseína. ${ }^{16}$ Uma possível influência da composição da fração protéica de fórmulas infantis e leite na bioacessibilidade do Ca tem sido relatada por vários autores. ${ }^{7,24,29,32} \mathrm{O}$ efeito favorável da caseína sobre a acessibilidade de Ca tem sido atribuído à inibição de precipitação do Ca por fosfato no lúmen intestinal pela ação de fosfopeptídeos provenientes da hidrólise enzimática da caseína, que mantém o Ca em uma forma solúvel e absorvível. ${ }^{12} \mathrm{O}$ Ca dialisável é maior nas fórmulas em que caseína é a principal fração protéica e no hidrolisado de proteínas do que nas outras fórmulas. ${ }^{7}$

Dessa forma, em relação aos resultados obtidos para o Ca bioacessível, utilizando método in vitro e diálise, pode-se observar grande similaridade em relação aos obtidos na literatura que utilizaram esta mesma metodologia, principalmente para as amostras de leite bovino integral in natura, leite de cabra integral UHT e extratos de soja.

Pode-se verificar que os resultados obtidos para $\mathrm{P}$ dialisado foram pouco maiores em relação ao Ca bioacessível, com exceção ao leite de ovelha, que apresentou resultado similar. Em geral, esse comportamento pode ser explicado, pois o $\mathrm{P}$ está intimamente associado ao $\mathrm{Ca}$ na nutrição humana, sendo que a proporção $\mathrm{Ca} / \mathrm{P}$ deve se apresentar em torno de 1:1, para manter o equilíbrio normal sérico. ${ }^{3}$

Os resultados para leite bovino integral in natura, bovino integral UHT e de cabra integral UHT também foram próximos, apresentando destaque para leite de cabra. Estudo sobre a presença de minerais nas frações solúveis, em que os elementos podem ser bem absorvidos, mostraram que para leite de cabra, 38,6\% de P estava nesta fração, similar ao aqui observado. No entanto, para o leite de ovelha, os resultados se mostraram diferentes. ${ }^{25}$

Nos alimentos de origem animal, o P é encontrado principalmente como composto orgânico e quando hidrolisado no trato gastrintestinal, libera fósforo inorgânico, que fica disponível para absorção. ${ }^{33}$ Indivíduos que consomem altas quantidades de produtos derivados do leite terão ingestão mais elevada de $\mathrm{P}$, uma vez que a densidade do P no leite bovino é superior ao observado em grande parte dos alimentos em uma dieta normal. ${ }^{3}$

A caseína é a principal proteína do leite e seus resíduos de serina são fosforilados. Nesses grupos, fosfatos ligam-se aos íons Ca, que ajudam a manter a estabilidade do complexo. Cerca de $30 \%$ do fosfato no leite bovino ocorre na forma livre, como fosfato inorgânico..$^{3,10}$

Essas informações da literatura ajudam a confirmar os resultados obtidos com os estudos in vitro para o leite bovino, isto é, $30,3 \pm 0,4 \%$ e $32 \pm 1 \%$ de bioacessibilidade de P para leite bovino in natura e UHT, respectivamente. Além disso, pode-se concluir que a fração bioacessível se encontre como P inorgânico.

Em bebês, tanto a quantidade de $\mathrm{P}$ ingerida quanto sua bioacessibilidade variam de acordo com a alimentação. Leite materno possui de 85 a $90 \%$ como teor bioacessível, seguido do leite de vaca (72\%), sendo que esses valores são menores em extratos de soja (59\%) em função da presença de ácido fítico. ${ }^{3}$ Comparando com os resultados obtidos no presente trabalho em relação ao extrato de soja, apesar de não ser possível fazer uma comparação com os valores obtidos nesse estudo em bebês, é possível concluir que também ocorre uma menor bioacessibilidade do $\mathrm{P}(20,0 \pm 0,4 \%)$ para o extrato de soja em relação às outras amostras.

\section{CONCLUSÃO}

Os teores totais de $\mathrm{Zn}$, Ca e $\mathrm{P}$ encontrados foram comparáveis aos de outros trabalhos descritos na literatura. Os estudos dos elementos dialisáveis, por sua vez, para determinar a tendência em relação à bioacessibilidade relativa para valores de $\mathrm{Zn}, \mathrm{Ca}$ e $\mathrm{P}$ a partir de diferentes amostras estudadas, apresentaram-se adequados, pois evitam a complexidade dos estudos in vivo.

Pode-se concluir que em $250 \mathrm{~mL}$ de leite (um copo) o organismo pode absorver cerca de 1,2 $\pm 0,2 \mathrm{mg}, 14,8 \pm 1,2 \mathrm{mg}, 4,5 \pm 0,8 \mathrm{mg}$, 2,2 $\pm 0,4 \mathrm{mg}$ e 4,9 $\pm 0,6 \mathrm{mg}$ de leite de ovelha in natura, soja, bovino in natura, cabra UHT e bovino UHT, respectivamente, para o elemento Zn. Já para $\mathrm{Ca}$, com a ingestão de um copo de leite temos $2079 \pm 133 \mathrm{mg}, 305 \pm 4 \mathrm{mg}, 2396 \pm 16 \mathrm{mg}, 2444 \pm 54 \mathrm{mg}$, $1527 \pm 71 \mathrm{mg}$ para leite de ovelha in natura, soja, bovino in natura, cabra UHT e bovino UHT, respectivamente. E para P o organismo 
pode absorver com a ingestão de um copo cerca de $2023 \pm 275 \mathrm{mg}$, $811 \pm 14 \mathrm{mg}, 2688 \pm 39 \mathrm{mg}, 5089 \pm 474 \mathrm{mg}$ e $3467 \pm 63 \mathrm{mg}$ para leite de ovelha in natura, soja, bovino in natura, cabra UHT e bovino UHT, respectivamente. Todos esses resultados de ingestão de um copo de leite estão apresentados em relação aos teores totais de Zn, Ca e P.

Os valores de ingestão diária recomendada na dieta humana para $\mathrm{Zn}$, Ca e P são 11, 1300 e $1250 \mathrm{mg}$ por dia, respectivamente. Os resultados apresentados neste trabalho correspondem aproximadamente 45, 118 e $267 \%$ dessa ingestão diária recomendada para Zn, Ca e P, respectivamente, se por exemplo, for ingerido um copo de $250 \mathrm{~mL}$ de leite bovino UHT.

Assim, os procedimentos in vitro possibilitam uma estimativa preliminar da bioacessibilidade dos nutrientes minerais, contribuindo de maneira positiva na definição de dietas.

\section{AGRADECIMENTOS}

À Fundação de Amparo à Pesquisa do Estado de São Paulo (FAPESP) (FAPESP no 06 / 59537-0, 2018/26145-9), ao Conselho Nacional de Desenvolvimento Científico e Tecnológico (CNPq) (processo 141846/2010-0), à Coordenação de Aperfeiçoamento de Pessoal de Nível Superior (CAPES) (processo 0001) pelo auxílio financeiro.

\section{REFERÊNCIAS}

1. Benito, P.; Miller, D.; Nutr. Res. 1998, 18, 581.

2. Fernández-García, E.; Carvajal-Lérida, I.; Pérez-Gálvez, A.; Nutr. Res. 2009, 29, 751 .

3. Cozzolino, S. M. F.; Biodisponibilidade de Nutrientes, Editora Manole Ltda.: Barueri, 2005.

4. Hodgkinson, A. J.; Wallace, O. A. M.; Smolenski, G.; Prosser, C. G.; Food Chem. 2019, 276, 619.

5. Gomez, B. G.; Perez-Corona M. T.; Madrid, Y.; J. Dairy Sci. 2016, 99, 9405.

6. Velasco-Reynold, C.; Navarro-Alarcon, M.; López-g de La Serrana, H.; Perez-Valero, V.; Lopez-Martinez, M. C.; Nutrition 2008, 24, 84.

7. Roig, M. J.; Alegría, A.; Barberá, R.; Farré, R.; Lagarda, M. J.; Food Chem. 1999a, 64, 403.

8. Cámara, F.; Amaro, M. A.; Barbera, R.; Clemente, G.; Food Chem. 2005, 92,481 .
9. Miquel, E.; Farré, R.; Trends Food Sci. Tech. 2007, 18, 139.

10. Yu, Y.; Qi, Y.; Jin, Y.; Food Biosci. 2019, 28, 143.

11. Silva, M. S.; Sele, V.; Sloth, J. J.; Araujo, P.; Amlund, H.; J. Chromatogr. B 2019, 1104, 262.

12. Miller, D. D.; Schricker, B. R.; Rasmussen Van Campen, D.; Am. J. Clin. Nutr. 1981, 34, 2248

13. Muniz-Naveiro, O.; Domínguez-González, R.; Bermejo-Barrera, A.; Bermejo-Barrera, P.; Cocho, J. A.; Fraga, J. M.; Anal. Bioanal. Chem. 2006, 385, 189 .

14. Buldini, P. L.; Cavalli, S.; Sharma, J. L.; Microchem. J. 2002, 72, 277.

15. Silva, F. V.; Lopes, G. S.; Nóbrega, J. A.; Souza, G. B.; Nogueira, A. R. A.; Spectrochim. Acta, Part B 2001, 56, 1909.

16. Gaucheron, F.; Reprod. Nutr. Dev. 2005, 45, 473.

17. Raynal-Ljutovac, K.; Lagriffoul, G.; Paccard, P.; Guillet, I.; Chilliard, Y.; Small Ruminant Res. 2008, 79, 57.

18. Penha, L. A. O.; Fonseca, I. C. B.; Mandarino, J. M.; Benassi, V. T.; Bol. Cent. Pesqui. Process. Aliment. 2007, 25, 91.

19. Momcilovic, B.; Belonje, B.; Giroux, A.; Shah, B. G.; J. Nutr. 1978, 106 , 913.

20. Scheplyagina, L. A.; J. Trace Elem. Med. Biol. 2005, 19, 29.

21. Michalke, B.; Ecotoxicol. Environ. Saf. 2003, 56, 122.

22. Thomsen, V.; Roberts, G.; Burgess, K.; Spectroscopy 2000, 15, 33.

23. Pereira Junior, J. B.; Fernandes, K. G.; Müller, R. C. S.; Nóbrega, J. A.; Palheta, D. C.; Quim. Nova 2009, 32, 2333.

24. Perales, S.; Barberá, R.; Lagarda, J.; Farré, R.; J. Agric. Food Chem. 2006, 54, 4901.

25. De La Fuente, M. A.; Olano, A.; Juárez, M.; Lait 1997, 77, 515.

26. Shen, L.; Robberecht, H.; Van Dael, P.; Deelstra, H.; Biol. Trace Elem. Res. 1995, 49, 107

27. Harzer, G.; Kauer, H. B. S.; Am. J. Clin. Nutr. 1982, 35, 981.

28. Pabón, M. L.; Lönnerdal, B.; J. Trace Elements Med. Biol. 2000, 14, 146.

29. Jovaní, M.; Barberá, R.; Farré, R.; Food Sci. Tech. Int. 2001, 7, 191.

30. Lonnerdal, B.; Cederblad, A.; Davidsson, L.; Sandstrom B.; Am. J. Clin. Nutr. 1984, 40, 1064

31. Johnson, P. E.; Evans, G. W.; Am. J. Clin. Nutr. 1978, 31, 416

32. Roig, M. J.; Alegría, R.; Barberá, R.; Farré, R.; Lagarda, M. J.; Food Chem. 1999b, 65, 353.

33. Shapiro, A. R.; Heaney, R. P.; Bone 2003, 32, 532. 